\title{
How to cope with negative estimates of components of variance in ecological field studies
}

\author{
D.J. Fletcher ${ }^{\mathrm{a}}$, A.J. Underwood ${ }^{\mathrm{b}, *}$ \\ ${ }^{a}$ Department of Mathematics and Statistics, University of Otago, Dunedin, New Zealand \\ ${ }^{\mathrm{b}}$ Centre for Research on Ecological Impacts of Coastal Cities, Marine Ecology Laboratories, A11, \\ University of Sydney, Sydney, NSW 2006, Australia
}

Received 6 September 2001; received in revised form 25 March 2002; accepted 2 April 2002

\begin{abstract}
Ecologists often need to estimate components of spatial or temporal variation. The most widely used method in ecology uses the observed and expected mean squares in an analysis of variance. A more general approach, which can be used for balanced and unbalanced designs, is based on residual maximal likelihood (REML). This method is less well known by ecologists and requires specialist software. If the design is balanced, the two methods are equivalent, except for one important respect: estimates from analysis of variance can be negative whereas REML estimates cannot. The purpose of this note is to point out a simple modification to the analyses of variance which yields the same estimates as REML for many of the designs commonly used in ecological studies. This modification has been available in the mathematical literature for over 30 years, but appears not to be well known amongst ecologists. It is useful in many cases of balanced analytical designs. (C) 2002 Published by Elsevier Science B.V.
\end{abstract}

Keywords: Analysis of variance; Hierarchical analysis; Spatial pattern; Variance components

\section{Introduction}

There are three broad reasons why ecologists wish or need to estimate the components of variation or magnitudes of treatment effects in their data - particularly where the data have come from long-term studies or are from coherently designed experimental analyses.

\footnotetext{
* Corresponding author. Tel.: +61-2-9351-2590; fax: +61-2-9351-6713.

E-mail address: aju@bio.usyd.edu.au (A.J. Underwood).
} 
Ecological studies are often designed to solve problems or to describe processes that operate at one or more spatial or temporal scales. Determining the relevant scales is not easy and may depend on a variety of approaches to sampling (e.g., Andrew and Mapstone, 1987). One useful method where the natural history of the system does not provide many clues about relevant scales is to use a nested hierarchical sampling design (see, for example, Morrisey et al., 1992). Having taken samples at a series of nested spatial scales, it is important to be able to determine where and at what scales there are variations in abundances, sizes, etc., of the organisms, so that further study is well focussed on the scales at which processes are creating patterns of difference. Under these circumstances, it will be necessary to separate the different spatial scales so that variation in the measurement of interest (e.g., density) can be independently determined at each of the chosen scales (see examples in Underwood and Chapman, 1996). Estimation of the components of variation at each scale therefore directly translates to the estimation of spatial variation at each spatial scale.

Such requirements are not just appropriate for studies involving spatial variation. As pointed out by Underwood $(1993,1994 \mathrm{a}, \mathrm{b})$, it is impossible to interpret temporal differences in, say, abundances of animals or plants unless there has been appropriate replication of temporal sampling to unconfound the differences from one time to another from those measured by spatial sampling at each time. Thus, to determine differences from one season to another requires that more than one independent time of sampling be examined in each season. Otherwise, however many replicate samples are taken at each time, the variation associated with the estimated mean in each season is spatial, not temporal variance.

Under these circumstances, estimation of temporal change needs to be separated from variation between replicate sample units at each time. Appropriate procedures for doing this were described in an ecological example by Kennelly and Underwood (1992) in a comparison of rates of change of fauna and flora in kelp-beds. The topic has been addressed again by Stewart-Oaten et al. (1995), who made the same point and provided some considerations of procedures to estimate variation associated with estimates of temporal variance.

The second reason for wishing to be able to compare the magnitudes of effects of different treatments is the desire to compare the relative magnitude of importance of different processes that affect ecological measures. For example, in areas where the densities of a species are regulated by processes of competition for resources and predation, it may be appropriate or necessary to determine the relative strengths or importances of these processes. Alternatively, there has been considerable debate about the relative importance of competitive interactions among different types of organisms (Connell, 1983; Schoener, 1983), which will not be resolvable until measures of the relative importance of competition are available for a wide variety of experimental studies.

A procedure for comparing the relative importance of different ecological processes was proposed by Welden and Slauson (1986) and has been widely cited in the ecological literature. It depends on assessing the proportional contribution to total variability in some measure that can be associated with each of the processes under consideration. The contributions are calculated as the sums of squares in an analysis of 
variance of the data under different combinations of experimental treatments. This method is seriously flawed in its logic and application (Underwood and Petraitis, 1993) and should not be used. Instead, more traditional uses of estimates of magnitudes of treatment effects calculated from mean squares are appropriate (see Burdick and Graybill, 1992; and Searle et al., 1992 for details). Such estimates are the focus of this paper.

A third and final reason for wishing to estimate the magnitudes of influences on ecological variables is the desire to compare studies from one place or time to another. Thus, there has been debate about the relative importance of ecological processes in one habitat (or region) or under particular circumstances compared with what happens in other habitats (or regions or circumstances). One method is to assess the contributions of processes in a series of studies, by inductive attempts to generalize from the particulars of the individual studies (e.g., Connell, 1983; Schoener, 1983; Sih et al., 1985; Menge and Farrell, 1989). Such syntheses can be aided by formal meta-analyses (Gurevitch et al., 1992), but would probably be more realistic if the studies being compared were designed with the specific intention of making such comparisons (Underwood and Petraitis, 1993). Either way, the relative importance of a process in one habitat can be compared with that in another habitat if there are reliable and independent estimates of the magnitude of the effects of that process on the variable being measured. Such estimates must be independent of the influences of other processes (e.g., the influence of predation must be separable from the effects of competition) and from the residual variation due to uncontrollable processes operating differently in different replicates of the experimental treatments. Again, therefore, the approach to be used involves estimating magnitudes of effects from analyses of variance of the data.

Given the widespread needs for such estimation in ecological studies, it is important to have tools that are readily understood that can help with the calculations. A well-known, easy-to-use and robust method for estimating components of variance uses the observed mean squares in the analysis of variance to estimate terms identified in the expected mean squares. For balanced designs, this method is simple to apply and involves an analysis already familiar to ecologists and environmental scientists. Alternative methods of estimation have been put forward in the statistical literature. An excellent summary of the theory underlying these methods was given by Searle et al. (1992). One method that has been generally accepted as having good properties for balanced and unbalanced designs is based on residual maximal likelihood (the REML method; see Robinson, 1987). This method is well known to applied statisticians and is available in some computer packages (e.g., SAS and Genstat).

It is known that in the case of balanced designs, analysis of variance and REML are equivalent apart from an important special case. This occurs when one or more of the estimates from analysis of variance are negative, leading to difficulty in their interpretation. This will occur, for example, when the variability at one spatial scale is less than would be predicted from the variability observed on a smaller scale. The REML estimates are defined in such a way that they can never be negative.

The purpose of this paper is to point out a simple modification to analysis of variance that makes it equivalent to REML when a balanced hierarchical design is used. The motivation for doing this is twofold. First, it allows ecologists to use a 
Table 1

Mean squares and estimates of the components of variance from the analysis of variance of the four levels of spatial hierarchy in sampling intertidal barnacles (see text for details)

\begin{tabular}{lrrr}
\hline Source of variation & $d f$ & Mean square & $\begin{array}{c}\text { Estimates of } \\
\text { spatial variation }\end{array}$ \\
\hline Among locations & 3 & 34,898 & -2711 \\
Among sites & 4 & 170,446 & 6182 \\
Among quadrats & 32 & 15,898 & 1805 \\
Among sub-quadrats & 160 & 6875 & 6875 \\
\hline
\end{tabular}

method that is already familiar and avoids the need for specialist software. Second, commonly, negative estimates from analysis of variance are automatically set to zero without consideration of the appropriateness of this procedure in terms of consequences for altering the remaining estimates. This leads to estimates that are not equivalent to those produced by the REML method and are generally not unbiased nor robust estimates.

The modification described here was first described by Thompson (1962), but appears not to be well known, even among applied statisticians. For the interested reader, a less mathematically oriented account of this work is to be found in Thompson and Moore (1963). Table 4 of that paper indicates the types of designs for which this procedure will lead to the same estimates as those from REML. The important point here is that such designs include all balanced hierarchical designs.

\section{Method and example}

Thompson and Moore (1963) described the procedure they called "pool-the-minimumviolator". For hierarchical designs, this procedure involves the following steps.

1. Calculate the estimates from analysis of variance.

2. If an estimate is negative, set it to zero, remove the corresponding factor from the model and re-calculate the estimates for the remaining factors. If there is more than one negative estimate, carry out this step for the factor with the smallest mean square only and go to Step 1.

To illustrate this method, consider the following analysis of densities of a barnacle (Chamaesipho tasmanica) on intertidal rocky shores on the coast of New South Wales, Australia. There were four spatial scales in the sampling scheme: four locations, parts of

Table 2

Mean squares and estimates of the components of variance from the analysis of variance of four levels of spatial hierarchy in sampling intertidal barnacles (as in Table 1) after removing the locations source of variation

\begin{tabular}{lrrl}
\hline Source of variation & $d f$ & Mean square & $\begin{array}{l}\text { Estimates of } \\
\text { spatial variation }\end{array}$ \\
\hline Among sites & 7 & 112,354 & 3858 \\
Among quadrats & 32 & 15,898 & 1805 \\
Among sub-quadrats & 160 & 6875 & 6875 \\
\hline
\end{tabular}


Table 3

Mean squares and estimates of the components of variance from the analysis of variance (as in Tables 1 and 2) and the modified estimates after removing two sources of variation that gave negative estimates of variation

\begin{tabular}{lrrrrrr}
\hline Source of variation & $d f$ & Mean square & $\begin{array}{l}\text { ANOVA } \\
\text { estimates }\end{array}$ & $\begin{array}{l}\text { Intermediate } \\
\text { estimates }\end{array}$ & $\begin{array}{l}\text { Final } \\
d f\end{array}$ & $\begin{array}{l}\text { Final } \\
\text { estimates }\end{array}$ \\
\hline Among locations & 3 & 34,898 & -2711 & -2711 & & 4226 \\
Among sites & 4 & 170,446 & 6582 & 6549 & 7 & 6712 \\
Among quadrats & 32 & 5898 & -195 & & 6712 & 192 \\
Among sub-quadrats & 160 & 6875 & 6875 & 672 & \\
\hline
\end{tabular}

the coast tens to hundreds of $\mathrm{km}$ apart; two sites tens to hundreds of metres apart were randomly chosen in each location; five quadrats some metres apart and five sub-quadrats $\left(20-25 \mathrm{~cm}^{2}\right)$ within each quadrat. In each sub-quadrat, the number of barnacles was recorded. The mean squares and usual estimates of the components of variance are shown in Table 1.

The negative estimate for variation among locations suggests that the variability between locations is smaller than would be expected from that observed at the smaller scales. Setting the estimate for that component to zero and removing Locations from the model, led to the mean squares in Table 2 . Note that the only change was for Sites, which now has $3+4=7 d f$ and a mean square given by:

$$
\{(3 \times 34,898)+(4 \times 170,446)\} /(3+4)=112,354 .
$$

No more steps are needed because the estimates for the remaining three factors were all positive. These estimates are exactly the same as would have been found using REML on a model with all four components of variance.

To illustrate an example where more than one estimate is negative, suppose the mean square for quadrats had been 5898. In this case, the estimates for locations and for quadrats are both negative (Table 3). Because the mean square for differences among quadrats is smaller, its estimate was set to zero and the variation among quadrats removed from the model. This gave the intermediate estimates in Table 3 . There is now just one negative estimate (among locations). This was also set to zero and that source of variation removed from the model, giving the final estimates shown in the table.

\section{Discussion}

Negative estimates of components of variance may occur because one or more of the assumptions associated with analysis of variance have been violated. This might occur, for example, if the variability between quadrats varies markedly from site to site. Hocking et al. (1989) advocated an approach to estimating components of variance that allows the suitability of the analysis of variance model to be assessed. In a similar vein, Smith and Murray (1984) proposed an alternative to the usual analysis in which observations are allowed to be correlated. Searle et al. (1992, pp. 61-62) provided a useful discussion of such ideas. The emphasis here has been to consider a simple method for producing reliable estimates, assuming that analysis of variance is reasonable. 
In some circumstances, it may be appropriate to remove terms in an order set by a priori hypotheses. For example, because of known ecological processes of recruitment or mortality of a species, it may be anticipated that there will be no variation in numbers at some spatial scales. Then, discovery of negative estimates at these scales would prompt them being removed, regardless of their relative size and which is the smallest. In the method as originally described, when there are more than one negative estimate, the smallest is removed first. This is for theoretical reasons described by Thompson and Moore (1963). Removal of a term can make a term higher in the table change from positive to negative or vice versa. Therefore, removing more than one term at a time or deciding to remove terms in some order set by hypotheses imposes a need for caution in the ensuing use of remaining estimates. In other words, removing two terms simultaneously because they are negative may be a mistake if one of them would have become positive if only the other had been removed. Provided care goes into the construction of the relevant hypotheses and the interpretations of the remaining estimates, no harm will be done in choosing the order to remove terms.

It should be noted that Thompson's (1962) method is quite different from simply setting any negative estimates from analysis of variance to zero, as can be seen by comparing the two types of estimate for sites and sub-quadrats in Table 3. It is hoped that ecologists will continue to be concerned with analyses of spatial and temporal variation and will take advantage of methods to help increase the usefulness of the estimates they make. By using a simple modification of familiar methods of analysis, the practice of setting estimates to zero and ignoring the consequences should become less prevalent.

\section{Acknowledgements}

We are grateful to Dr K.R. Clarke and an anonymous referee for their comments on the paper and to Dr Gee Chapman for discussions on this topic and for use of the example cited. A.J.U.'s contribution was supported by a grant from the Australian Research Council and from the Centre for Research on Ecological Impacts of Coastal Cities; DJF was supported by funds from the Institute of Marine Ecology, University of Sydney.[RW]

\section{References}

Andrew, N.L., Mapstone, B.D., 1987. Sampling and the description of spatial pattern in marine ecology. Annu. Rev. Oceanogr. Mar. Biol. 25, 39-90.

Burdick, R.K., Graybill, F.A., 1992. Confidence Intervals on Variance Components. Marcel Dekker, New York.

Connell, J.H., 1983. On the prevalence and relative importance of interspecific competition: evidence from field experiments. Am. Nat. 122, 661-696.

Gurevitch, J., Morrow, L.L., Wallace, A., Walsh, J.S., 1992. A meta-analysis of competition in field experiments. Am. Nat. 140, 539-572.

Hocking, R.R., Green, J.W., Bremer, R.H., 1989. Variance-component estimation with model-based diagnostics. Technometrics 31, 227-239.

Kennelly, S.J., Underwood, A.J., 1992. Fluctuations in the distributions and abundances of species in sublittoral kelp forests in New South Wales. Aust. J. Ecol. 17, 367-382.

Menge, B.A., Farrell, T.M., 1989. Community structure and interaction webs in shallow marine hard-bottom communities: tests of an environmental stress model. Adv. Ecol. Res. 19, 189-262. 
Morrisey, D.J., Howitt, L., Underwood, A.J., Stark, J.S., 1992. Spatial variation in soft-sediment benthos. Mar. Ecol. Progr. Ser. 81, 197-204.

Robinson, D.L., 1987. Estimation and use of variance components. The Statistician 36, 3-14.

Schoener, T.W., 1983. Field experiments on intraspecific competition. Am. Nat. 122, 240-285.

Searle, S.R., Cassella, G., McCulloch, C., 1992. Variance Components. Wiley, New York.

Sih, A., Crowley, M., McPeek, M., Petranka, J., Strohmeier, K., 1985. Predation, competition, and prey communities: a review of field experiments. Annu. Rev. Ecol. Syst. 16, 269-311.

Smith, D.W., Murray, L.W., 1984. An alternative to Eisenhart's model II and mixed model in the case of negative variance estimates. J. Am. Stat. Assoc. 79, 145-151.

Stewart-Oaten, A., Murdoch, W.W., Walde, S.J., 1995. Estimation of temporal variability in populations. Am. Nat. 146, 519-535.

Thompson, W.A., 1962. The problem of negative estimates of variance components. Ann. Math. Stat. 33, 273289.

Thompson, W.A., Moore, J.R., 1963. Non-negative estimates of variance components. Technometrics 5, 441449.

Underwood, A.J., 1993. The mechanics of spatially replicated sampling programmes to detect environmental impacts in a variable world. Aust. J. Ecol. 18, 99-116.

Underwood, A.J., 1994a. On beyond BACI: sampling designs that might reliably detect environmental disturbances. Ecol. Appl. 4, 3-15.

Underwood, A.J., 1994b. Spatial and temporal problems with monitoring. In: Calow, P., Petts, G.E. (Eds.), Rivers Handbook, vol. 2. Blackwell, London, pp. 101-123.

Underwood, A.J., Chapman, M.G., 1996. Scales of spatial patterns of distribution of intertidal snails. Oecologia $107,212-224$.

Underwood, A.J., Petraitis, P.S., 1993. Structure of intertidal assemblages in different locations: how can local processes be compared? In: Ricklefs, R.E., Schluter, D. (Eds.), Species Diversity in Ecological Communities: Historical and Geographical Perspectives. Univ. of Chicago Press, Chicago, pp. 38-51.

Welden, C.W., Slauson, W.L., 1986. The intensity of competition versus its importance: an overlooked distinction and some implications. Q. Rev. Biol. 61, 23-44. 